INVESTIGACIÓN

Recibido: 12/08/2020 --- Aceptado: 14/12/2020 --- Publicado: 12/03/2021

\title{
COMUNICACIÓN Y ESPACIOS CULTURALES EN TIEMPOS DE COVID-19
}

\section{Communication and cultural spaces in times of COVID-19}

87 Inma García-Martín. Universidad de Salamanca. España. adagarcia@usal.es

Félix Ortega-Mohedano. Universidad de Salamanca. España. fortega@usal.es

María-Esther Pérez-Peláez. Universidad Internacional de Valencia. España. mariaesther.perez@campusviu.es

\section{RESUMEN}

El COVID-19 ha ocasionado el cierre de los grandes entes culturales que ha impactado en los hábitos culturales de los ciudadanos. Desde el inicio de esta Pandemia Global, las herramientas digitales se han convertido en las grandes aliadas de los museos para seguir en contacto con sus visitantes, reforzando sus vínculos y apostando por la digitalización de contenidos tanto en formato directo como diferido. Analizar la eficacia de esta comunicación digital y los hábitos de uso y consumo que caracteriza a los visitantes de los museos para readaptar y mejorar las estrategias de comunicación de los museos en tiempos de Coronavirus es fundamental. Se trata de un estudio de carácter estadístico - descriptivo donde se presentan los resultados de un cuestionario realizado a 619 visitantes de dos de los museos con mayor número de visitas en Castilla y León: El Museo de La Evolución Humana y El Museo Art Nouveau y Art Decó Casa Lis. Entre los principales resultados destaca el aumento en el número de visitantes que accede a la información a través de redes sociales y servicios móviles, lo que implica un cambio en la forma de informarse, pero también se detecta la necesidad de implementar estrategias digitales para afrontar una crisis como el COVID-19.

PALABRAS CLAVE: COVID-19 - Coronavirus - Pandemias - Museos - Redes Sociales - Recursos digitales - Educación. 
COVID-19 has caused the closure of large cultural entities that has impacted on the cultural habits of citizens. Since the start of this Global Pandemic, digital tools have become the great allies of museums to keep in touch with their visitors, strengthening their links and betting on the digitization of content both in direct and delayed format. Analyzing the effectiveness of this digital communication and the habits of use and consumption that characterize museum visitors to readjust and improve museum communication strategies in times of Coronavirus is essential. This is a statisticaldescriptive study that presents the results of a questionnaire carried out on 619 visitors from two of the museums with the highest number of visits in Castille and León: The Museum of Human Evolution and The Art Nouveau and Art Deco Museum Casa Lis. Among the main results, the increase in the number of visitors who access information through social networks and mobile services stands out, which implies a change in the way they inform themselves, but the need to implement digital strategies to face a crisis like the COVID-19.

KEY WORDS: COVID-19 - Coronavirus - Pandemics - Museums - Social networks Digital resources - Education.

\section{COMUNICAÇÃO E ESPAÇOS CULTURAIS EM TEMPOS DE COVID-19}

\section{RESUMO}

A COVID-19 tem ocasionado o fechamento das grandes entidades culturais que tem impactado nos hábitos culturais dos cidadãos. Desde o começo desta Pandemia Global, as ferramentas digitais têm se transformado nas grandes aliadas dos museus para continuar em contato com os seus visitantes, reforçando os seus vínculos e apostando pela digitalização de conteúdos tanto em formato direto quanto diferido. Analisar a eficácia desta comunicação digital e os hábitos de uso e consumo que caracteriza aos visitantes dos museus para readaptar e melhorar as estratégias de comunicação dos museus em tempos de Coronavírus é fundamental. Se trata de um estudo de caráter estatístico - descritivo onde são apresentados os resultados de um questionário realizado a 619 visitantes dos museus com maior número de visitas em Castilla e León: O Museo de La Evolución Humana e O Museu Art Nouveau e Art Decó Casa Lis. Dentro dos principais resultados se destaca o aumento no número de visitantes que acessam a informação através de redes sociais e serviços móveis, o que implica uma mudança na forma de se manter informado, mas também se detecta a necessidade de implementar estratégias digitais para enfrentar uma crise como o COVID-19.

PALAVRAS CHAVE: COVID-19 - Coronavírus - Pandemias - Museus - Redes Sociais

- Recursos digitais - Educação.

\section{Cómo citar el artículo:}


García-Martín, I., Ortega-Mohedano, F. y Pérez-Peláez, M. E. (2021). Comunicación y espacios culturales en tiempos de COVID-19. Vivat Academia. Revista de Comunicación, 154, 21-43. http:// doi.org/10.15178/va.2021.154.e1261

http://www.vivatacademia.net/index.php/vivat/article/view/1261

\section{INTRODUCCIÓN}

El sector cultural es uno de los más afectados por el coronavirus (virus SARS-CoV2) y los museos no son una excepción. El Consejo Internacional de Museos (2020) reconoce "los múltiples desafíos a los que se enfrentan los museos y los profesionales de los museos durante este periodo" (ICOM, 2020).

El COVID-19 ha cambiado el día a día de la sociedad a pasos agigantados, también en el ámbito de la cultura. El cierre de los espacios culturales que lleva vigente desde mediados de marzo ha ocasionado que muchos de estos entes culturales hayan reforzado su presencia online, a través de herramientas digitales, como páginas web, redes sociales y contenido online. Este aislamiento forzado al que se han visto sometidos limita las posibilidades para los museos de llegar a su público, pero varias instituciones en todo el mundo están llevando a cabo iniciativas y mostrando una gran resiliencia aprovechando el poder de comunicación en redes sociales y las herramientas digitales.

En este artículo se presentan los resultados de una encuesta online a los visitantes de los museos de Castilla y León, sobre los hábitos de uso y consumo en el contexto de la comunicación, a través de las herramientas digitales y redes sociales, de los museos con su público. De esta forma, nos interesa analizar tanto el comportamiento como el impacto de las tecnologías en el público de estos museos en tiempos de COVID-19. Además, también se analizan una serie de estudios de caso y buenas prácticas en comunicación digital, desde la activación del estado de alarma, que pueden ser ejemplos inspiradores para otros museos.

\subsection{Revisión de la literatura}

La digitalización de las obras de arte y la comunicación online a través de redes sociales representan una gran oportunidad en la promoción del patrimonio, para reforzar la relación entre museo-visitante y, sobre todo, para fomentar los procesos de interacción con el público. Esta práctica de compartir las colecciones en línea se lleva desarrollando en los museos desde hace años, pero durante estas semanas de confinamiento, en todas las instituciones se ha intensificado la actividad en redes sociales $\mathrm{y}$, con ella, ha aumentado el seguimiento de la audiencia.

Tal y como señala Ayala, Cuenca-Amigo y Cuenca (2019) en el museo “los procesos de comunicación y las estrategias de marketing constituyen uno de los retos identificados" (p. 66). Por ello, en la actualidad es fundamental analizar la 
comunicación desarrollada en los museos, tanto la expuesta dentro, como la exterior del museo (redes sociales, medios de comunicación, carteles o folletos) que compromete a la visita de la audiencia (Mateos, 2012) y ha permitido conectar durante el contexto del COVID-19. Es importante resaltar que desde el 2005, la Unesco insta a las instituciones museísticas a ser conscientes de la importancia de las Tecnologías de la Información y la Comunicación (TIC) para preservar, educar, investigar y comunicar (Mariátegui, 2020).

Con anterioridad al nuevo contexto de la pandemia, la museología se encontraba inmersa en el desarrollo de programas sobre el uso de las herramientas digitales y, que puede apreciarse en museos nacionales, como señala Delgado (2018) en el caso del Museo Thyssen de Madrid. Para este museo la tecnología como soporte le ha permitido la digitalización de su contenido para hacerlo más accesible a todos los públicos y potenciar el museo digital a través de convenios con otras galerías internacionales.

Otro de los museos que ha incorporado la tecnología con éxito es el Museo del Prado. En este caso, han apostado por las aplicaciones móviles para completar las visitas durante las exposiciones temporales, a través de digitalizaciones de libros, creando zoom de alta definición sobre detalles de algunas obras o vistas $360^{\circ}$ sobre piezas conocidas (Pantoja, 2016). El uso de la tecnología abarca múltiples áreas dentro del museo, desde dar soporte en la comunicación y el marketing (Ayala, CuencaAmigo y Cuenca, 2019) hasta la interpretación de los contenidos a través de dispositivos digitales durante la visita (Cordón, 2012).

En primer lugar, Internet y el mundo online permiten que la comunicación y el marketing desarrollen campañas digitales muy eficaces para los museos (Marakos, 2014) y, además, hacen accesible el contenido de los mismos (Lorente, 2015). Se produce, por tanto, una comunicación bidireccional entre los museos y la audiencia gracias a las ventajas de la implantación de las tecnologías digitales en la gestión de las instituciones museísticas. En lo referente a los estudios de público y análisis de audiencias, durante los últimos tiempos se han desarrollado los sistemas Ticketing y CRM, que permiten generar y almacenar información sobre datos para una posterior toma de decisiones sobre el marketing cultural (Leal y Queno, 2011).

En segundo lugar, la revolución social y cultural generada por Internet se ha convertido en un imprescindible para el día a día de la sociedad. La audiencia espera y demanda nuevas actividades, que resulten interactivas y multidisciplinares. Ahora existen entornos interactivos y dinámicos, basados en el poder de la imagen, en su capacidad de atracción, capaces de generar participación por parte del espectador. Estos aspectos son los relacionados con el uso de la tecnología y la digitalización en el ámbito museístico (Black, 2012). Internet ha supuesto un gran avance para los museos, cada vez son más los que disponen de catálogos web, podcats con audioguías, dispositivos tecnológicos como pantallas táctiles, reconocimiento facial, geolocalización o códigos QR y apuestan por formar parte de iniciativas como Google Art Proyect (Cordón, 2012). Sin embargo, matiza este autor, los museos deben apostar 
por las posibilidades que Internet les ofrece, dentro de sus posibilidades, y de acuerdo a su línea estratégica de comunicación.

Pero Internet, como otras herramientas digitales, debe considerarse como un soporte para el museo físico, y nunca, como un sustituto (Almazán, 2007) y como defiende Rodríguez (2011) la tecnología dentro de un museo debe abogar por el impulso de la comunicación sin sobresalir sobre la museografía de la institución. De esta forma, la aplicación de estas herramientas dentro de los museos requiere de un análisis para su óptima puesta en marcha. En esta línea de actuación, cabe destacar como ejemplo de líder en aplicaciones tecnológicas al Museo de Arte Contemporáneo de Nueva York (MOMA) que cuenta con infinidad de canales multimedia, productos interactivos como son las exhibiciones especiales, conversaciones, blogs y descripciones visuales. Otro museo, también de esta ciudad, quizás no tan reconocido, que pone a disposición del público, de forma gratuita y digital, todas sus publicaciones y colecciones, es The New Museum. Por su parte, otro de los museos que ha destacado por su innovadora estrategia digital y es ejemplo de buenas prácticas es El Museo Nacional de Historia Natural (Smithsonian), ubicado en Washington, en el que, a través de códigos QR, los visitantes pueden comprobar cómo se verían sus rasgos en épocas pasadas.

De esta forma, se hace evidente que el interés en las nuevas tecnologías para la creación de contenidos y el desarrollo de aplicaciones digitales en museos ya estaba vigente al menos, como señalan algunos autores, desde hace cinco años antes de este nuevo contexto de COVID-19 (Delgado Pacheco, 2020). Durante la cuarentena, los principales entes del sector cultural elaboraron una serie de pautas para potenciar la comunicación virtual con el público (AMM, 2020a; AMM, 2020b; ICOM, 2020), urgiendo a los museos hacia un modelo virtual y accesible. Tal y como señala Delgado Pacheco (2020), un museo es más accesible si comparte libremente contenido y desarrolla programas didácticos a través de sus plataformas digitales.

Aun así, el estudio de las tecnologías y las aplicaciones digitales suponen un reto para los museos. Propulsar la difusión de las colecciones, exposiciones y la información sobre el acceso, con la finalidad de alcanzar tanto a los visitantes reales como a los virtuales, son algunas de las tendencias de estas instituciones culturales (Tamacas, 2016) y que va en consonancia con las recomendaciones en torno al COVID19. Es importante mencionar que el contexto de la pandemia se ha convertido en una oportunidad para los museos de impulsar su compromiso digital al verse forzados a reinventar sus estrategias digitales. Sin embargo, los primeros estudios sobre este contexto de crisis reportan una serie de problemas que atañen a los museos y que no están permitiendo el avance de las pautas señaladas ante esta nueva realidad. Una vez más, los museos adolecen de estudios de públicos, escasas estrategias de inclusión y falta de colaboración con otras instituciones (Holmquist, 2020 y Riofrío et al., 2019).

Asimismo, Delgado Pacheco (2020) afirma que, aunque existe trabajo previo de virtualización y conexión con el público a través de plataformas digitales, los museos que desarrollaron el uso de las TIC, enfrentan la crisis con mejores opciones de

Vivat Academia. Revista de Comunicación. 2021, nº 154, 21-43 
comunicación, conexión con sus audiencias y manejo de públicos. Además, otros estudios han detectado - durante la cuarentena - un incremento de visitas a contenidos culturales a través de dispositivos, como es el caso de los museos online como El Museo del Prado que ha popularizado sus vídeos en Instagram de una hora de duración diaria (Perez-Rufi, 2020).

En este contexto, el uso de las tecnologías digitales para la mejora de la experiencia de visita en el museo requiere de un profundo análisis debido, fundamentalmente, a la aparición de nuevos hábitos de consumo de información y que está modificando la forma en que los ciudadanos acceden a la información, también cultural. Por ello, es esencial estudiar los hábitos de uso y consumo, las opiniones y valoraciones de los visitantes respecto a la hora de obtener información e interactuar con los museos. La coyuntura del COVID-19 supone una gran oportunidad para estudiar la comunicación de los museos con su audiencia para salir reforzados o en su caso modificar las estrategias que no están teniendo los resultados esperados.

\section{OBJETIVOS}

El objetivo principal de esta investigación es analizar los hábitos de uso y consumo de los visitantes de los museos de Castilla y León. Para alcanzarlo se propone realizar un estudio de visitantes de dos museos referentes - el Museo de la Evolución Humana de Burgos y el Museo Art Nouveau y Art Déco de Salamanca -, los más importantes por visitantes de la CCAA de Castilla y León, conociendo así de forma directa los hábitos de uso y consumo digitales, su opinión sobre los procesos educomunicativos e interacción que experimentan en los espacios físicos y digitales en el contexto del COVID-19.

El objetivo general se concreta en los objetivos específicos que se exponen a continuación:

1. Conocer la relación existente entre el perfil del público visitante y las variables relacionadas con la utilización de dispositivos y redes sociales durante la visita.

2. Estudiar la relación existente entre el perfil del público visitante y las variables relacionadas con la persona y las preferencias.

3. Analizar la relación existente entre el perfil del público visitante y las variables relacionadas con la persona y la valoración de recursos digitales.

4. Conocer la relación existente entre el perfil del público visitante y las variables relacionadas con la persona y el aprendizaje durante la visita.

\section{METODOLOGÍA}

La investigación realizada es de tipo descriptivo ya que las variables estudiadas han sido medidas en un contexto natural y no han sido manipuladas. Para el estudio de los datos, se optó por un diseño de investigación transversal a través del diseño de una encuesta online tomando como referencia para la selección de la muestra los datos de media de visitantes de cada museo N, entre los años 2011 a 2017, 296.337 visitantes de 
media visitaron el Museo de la Evolución Humana de Burgos y 113.740 en el Museo de Art Decó y Art Nouveau-Casa Lis de Salamanca. El cuestionario se administró a una primera muestra piloto de 30 individuos de uno de los museos participantes que consintió corregir el cuestionario y sintetizar variables. Posteriormente, la encuesta fue realizada durante los meses de abril y mayo del año 2017 en la puerta de entrada de los dos museos - nB= 310 entrevistas válidas en Burgos y nS=309 en Salamanca -, asistida por un T-CAPI (Tablet and Computer Assisted Personal Interview) y por un equipo de 4 encuestadores previamente formados.

Específicamente, se procedió a ponderar la muestra utilizando la población total y distribución de Salamanca y Burgos, el 50\% de los visitantes son residentes en la provincia de ubicación del museo. La muestra aleatoria válida final fue de nT=619 visitantes entre los dos museos, con un nivel de confianza de un 95\% con un error muestral del $+-5.5 \%$ para poblaciones finitas en cada museo. Para el análisis de los datos, se llevaron a cabo distribuciones de frecuencias y representaciones gráficas de cada una de las preguntas del cuestionario $y$, por otra, se crearon tablas de contingencia para representar las asociaciones significativas entre variables mediante la prueba estadística Chi cuadrado $\left(\chi^{2}\right)$. Tanto para el análisis como cálculo de fiabilidad de los datos se empleó el programa estadístico SPPS y se contó con la participación de un experto investigador.

Tabla 1: Descripción de la muestra según la variable Edad.

\begin{tabular}{|c|c|c|}
\hline EDAD & $\mathbf{N}$ & $\%$ \\
\hline$\leq 24$ & 216 & 34,9 \\
\hline $25-44$ & 184 & 29,7 \\
\hline$\geq 45$ & 219 & 35,4 \\
\hline TOTAL & 619 & 100,0 \\
\hline
\end{tabular}

Fuente: Elaboración propia.

Con respecto a la variable Edad (V1) los dos grupos con mayor índice de visita a los museos son, por un lado, los mayores de 45 años $(\mathrm{N}=219 ; 35,4 \%) \mathrm{y}$, por otro lado, el grupo de visitantes menor de 24 años $(\mathrm{N}=216 ; 34,9 \%)$. El tercer tramo de edad lo representan los visitantes entre 25 y 44 años ( $\mathrm{N}=184 ; 29,7 \%)$.

Tabla 2: Descripción de la muestra según la variable Sexo.

\begin{tabular}{|c|c|c|}
\hline SEXO & N & $\%$ \\
\hline Hombre & 291 & 47,0 \\
\hline
\end{tabular}


García-Martín, R., Ortega-Mohedano, F. y Pérez-Peláez, M. E.

Comunicación y espacios culturales en tiempos de COVID-19

\begin{tabular}{|c|c|c|}
\hline Mujer & 328 & 53,0 \\
\hline TOTAL & 619 & 100,0 \\
\hline
\end{tabular}

Fuente: Elaboración propia.

Respecto de la variable Sexo, prácticamente la mitad de los visitantes son mujeres $(\mathrm{N}=328 ; 53 \%)$ y la otra mitad son hombres $(\mathrm{N}=291 ; 47 \%)$.

Tabla 3: Descripción de la muestra según la variable Nivel de Estudios.

\begin{tabular}{|c|c|c|}
\hline ESTUDIOS & N & $\%$ \\
\hline Primarios, EGB, ESO & 32 & 5,3 \\
\hline Bachillerato, COU, FP & 231 & 38,2 \\
\hline Universitarios & 342 & 56,5 \\
\hline Total & 605 & 97,7 \\
\hline Perdidos Sistema & 14 & 2,3 \\
\hline TOTAL & 619 & 100,0 \\
\hline
\end{tabular}

Fuente: Elaboración propia.

Respecto de la variable Nivel de Estudios, más de la mitad de los visitantes poseen estudios universitarios ( $\mathrm{N}=342 ; 56,5 \%)$. El segundo grupo de visitantes, pero con mucha distancia son personas con nivel de estudios secundarios ( $\mathrm{N}=231 ; 38,5 \%)$. Ha de destacarse la baja participación de personas con estudios primarios ( $N=32 ; 5,3 \%)$.

Tabla 4: Descripción de la muestra según la variable Procedencia.

\begin{tabular}{|c|c|c|}
\hline PROCEDENCIA & N & $\%$ \\
\hline Castilla y León & 377 & 60,9 \\
\hline Fuera de Castilla y León & 180 & 29,1 \\
\hline Extranjero & 62 & 10,0 \\
\hline TOTAL & 619 & 100,0 \\
\hline
\end{tabular}

Fuente: Elaboración propia. 
Respecto al Lugar de Procedencia, la mayoría del público visitante proviene de la propia comunidad ( $\mathrm{N}=377 ; 60,9 \%)$. En segundo lugar, se encuentran los visitantes del resto de España ( $\mathrm{N}=180 ; 29,1 \%)$. Por su parte, los visitantes extranjeros son grupo que menor porcentaje de visitas acumula $(\mathrm{N}=62 ; 10 \%)$.

\section{RESULTADOS}

\subsection{Utilización de dispositivos y redes sociales durante la visita}

El análisis del uso de dispositivos por parte del público visitante en función de la edad permite observar hábitos interesantes. Con un porcentaje de $12,5 \%$, se aprecia que los menores de 24 años son los que menos proporción tienen en Ningún dispositivo, o dicho de otro modo, los que con mayor frecuencia usan algún dispositivo. El siguiente tramo de edad que destaca por el uso de algún dispositivo es entre $25-44$ años $(21,7 \%)$, y por último, los mayores de 45 años tienen una porcentaje de $25,1 \%$.

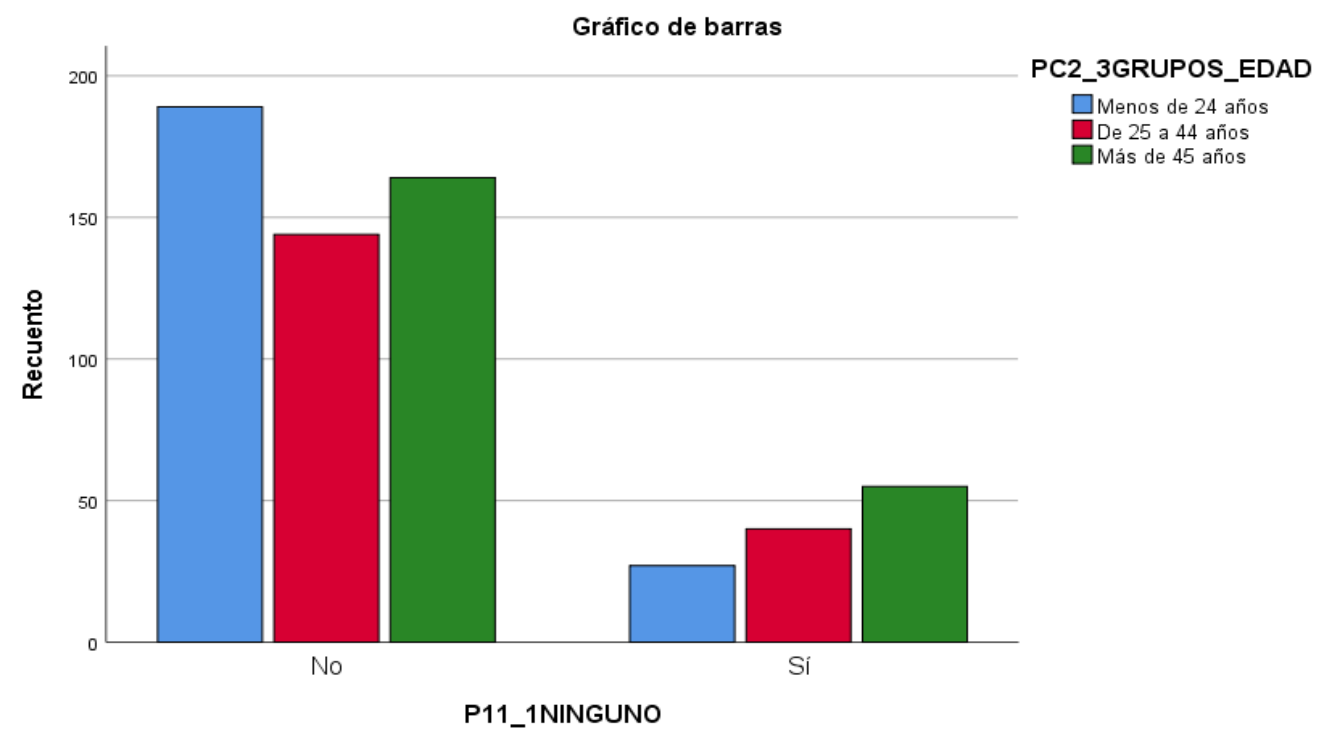

Gráfico 1: Histograma de la variable Ningún Dispositivo.

Fuente: Elaboración propia.

El 64,4\% del grupo de visitantes menor de 24 años utiliza una Tablet o teléfono durante la visita a los museos, el margen de edad mayor de 45 años tiene un porcentaje de $50,2 \%$ en uso de estos dispositivos, seguidos por el grupo de visitantes entre 25 a 44 años $(48,9 \%)$. Sin embargo, atendiendo a los desgloses por museos, existe una clara diferencia entre el Museo de Burgos, en el que un 85,5\% de los menores de 24 años afirman utilizar una Tablet o teléfono durante la visita, seguidos por el 75,3\% de los mayores de 45 años y el 70,7\% de entre 25-44 años. El Museo de Salamanca, obtiene menores frecuencias de uso, el 72,8\% entre 25-44 años dicen no usar estos dispositivos, seguidos del grupo mayor de 45 años (68,3\%) y los menores de 24 años (60,6\%). 


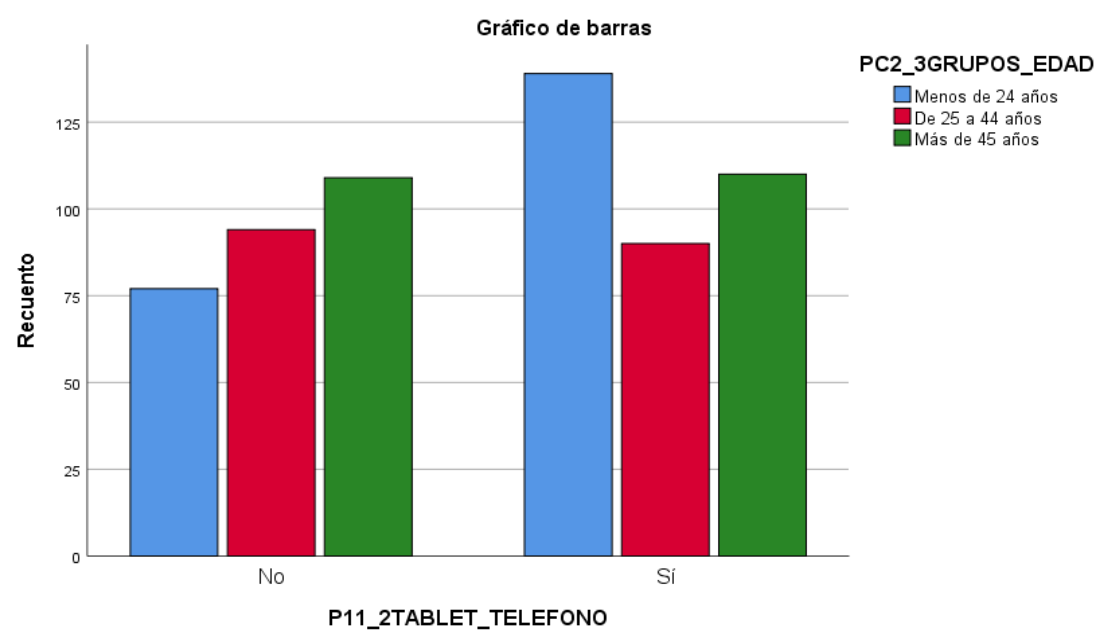

Gráfico 2: Histograma de la variable uso de Tablet o teléfono. Fuente: Elaboración propia.

En conclusión, los menores de 24 años son los que con mayor frecuencia usan algún dispositivo. Cuanto menor es la edad del visitante mayor es el uso de dispositivos. La Tablet y el teléfono inteligente son los dispositivos más utilizados durante la visita. Por su parte, el Museo Casa Lis de Salamanca destaca por el escaso uso de dispositivos durante la visita, quizás por la prohibición de realizar fotografías o vídeos dentro de las instalaciones.

Por su parte, atendiendo al uso de redes sociales en función de la edad podemos observar que de las personas que comparten una publicación del museo que visitan, el 32,6\% tiene entre 25-44 años, seguidos por los menores de 24 años (30,6\%) y los mayores de 45 años con un 16,9\%. Es decir, se aprecian bajas frecuencias de interacción con la acción compartir una publicación de los museos en las redes sociales.

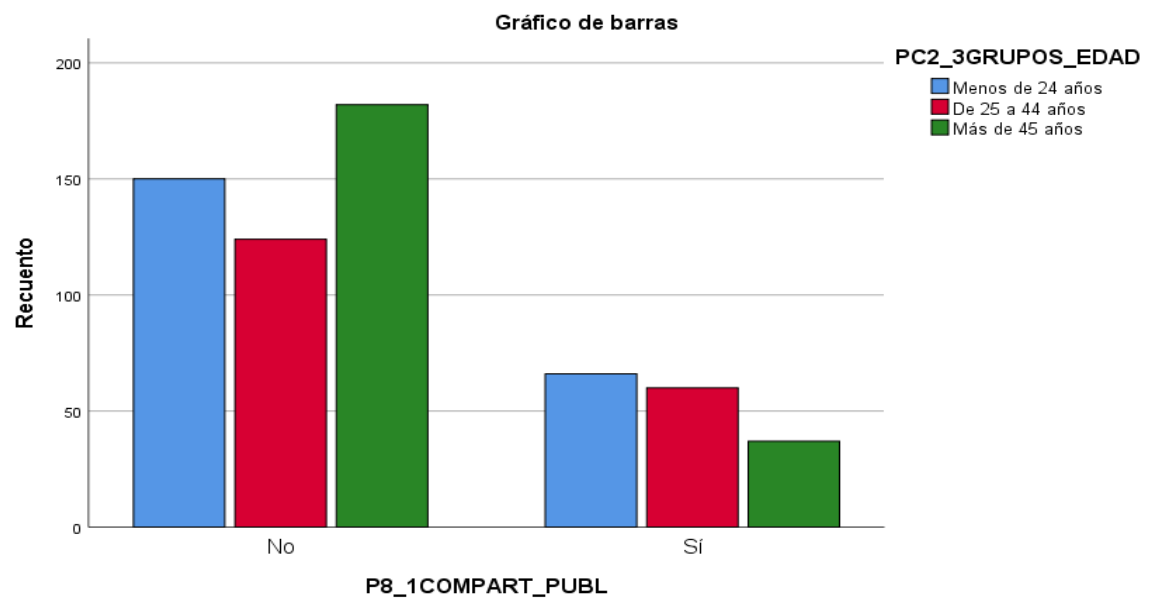

Gráfico 3: Histograma de la variable publicación en redes sociales.

Fuente: Elaboración propia. 
Por su parte, el 34,7\% de los visitantes mayores de 45 años afirman participar en un evento o juego del museo en redes sociales, el 10,9\% que participa es el grupo de 25-44 años, y el resto sólo el 6,9\% de los menores de 24 . Una vez más, son los mayores de 45 años los que presentan mayor participación en redes sociales, el 11,4\% menciona en redes sociales al museo que visitan, y sólo el 5,4\% de 25-44 años lo hace.

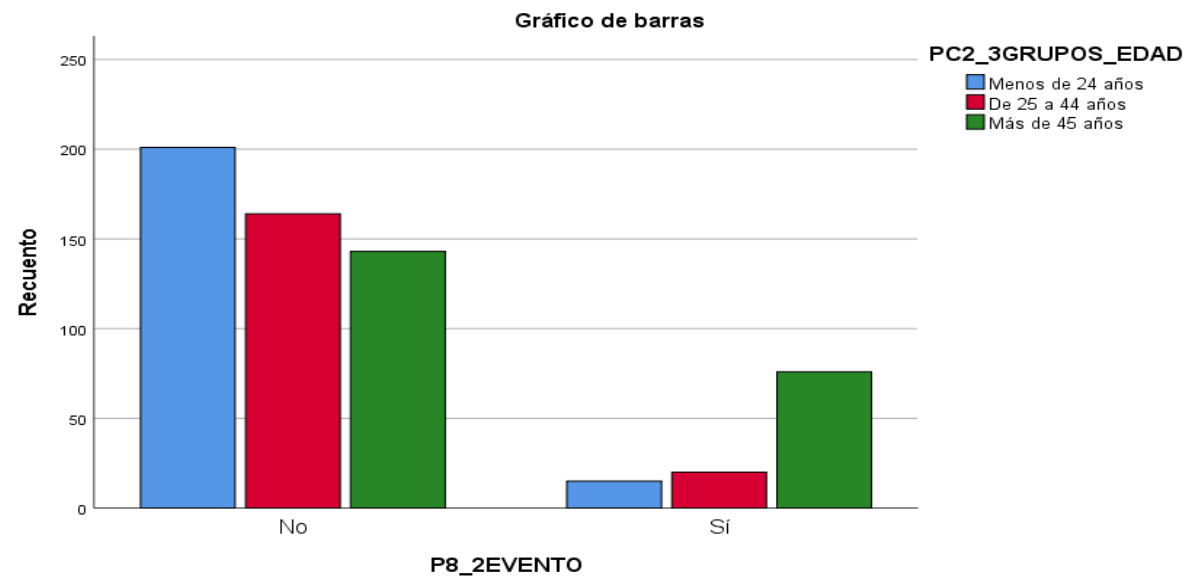

Gráfico 4: Histograma de la variable participar en evento o juego.

Fuente: Elaboración propia.

Si analizamos los resultados por museos, separadamente, las conclusiones pueden variar ligeramente. En el Museo de Salamanca, de los que publican fotos $(p=, 006)$, se aprecian diferencias significativas en el rango de edad comprendida entre 25-44 años, con un $16,3 \%$, son el grupo de edad que más/con mayor prevalencia publican fotografías de los museos que visitan.

En conclusión, según aumenta la edad del visitante, aumenta la interacción con las redes sociales del museo, sin embargo, aunque los mayores de 45 años muestran mayor frecuencia de uso de redes, la interacción con el museo presenta escasa prevalencia.

\subsection{Preferencias del público visitante en función de su perfil}

El análisis de las preferencias de los visitantes en función de la edad muestra que de los visitantes de la muestra que acceden a los museos entre 0 y 1 veces al año, el $55,1 \%$ tiene menos de 24 años, el 50,8\% entre 25 a 44 años y el 14,6\% más de 45 años. De los visitantes que visitan los museos de 2 a 4 veces al año, el 40,6\% tiene menos de 24 años, el 33,3\% entre 25 a 44 años y el 19,4\% más de 45 años. De los que afirman visitar los museos de 5 a 7 veces al año, el 1,4\% tiene menos de 24 años, el 4,8\% entre 25 a 44 años y el 5,6\% más de 45 años. De los visitantes aseguran visitar los museos más de 8 veces al año, el 2,9\% tiene menos de 24 años, el 11,1\% entre 25 a 44 años y el $60,4 \%$ más de 45 años.

Tabla 5: Descripción de la muestra según la frecuencia de visita 
García-Martín, R., Ortega-Mohedano, F. y Pérez-Peláez, M. E. Comunicación y espacios culturales en tiempos de COVID-19

en función de la edad.

\begin{tabular}{|c|c|c|c|}
\hline FRECUENCIA & $\begin{array}{c}\text { Menos de 24 } \\
\text { años }\end{array}$ & De 25 a 44 años & Más de 45 años \\
\hline De 0 a 1 veces al año & $55,1 \%$ & $50,8 \%$ & $14,6 \%$ \\
\hline De 2 a 4 veces al año & $40,6 \%$ & $33,3 \%$ & $19,4 \%$ \\
\hline De 5 a 7 veces al año & $1,4 \%$ & $4,8 \%$ & $5,6 \%$ \\
\hline Más de 8 veces al año & $2,9 \%$ & $11,1 \%$ & $60,4 \%$ \\
\hline TOTAL & $100,00 \%$ & $100,00 \%$ & $100,00 \%$ \\
\hline
\end{tabular}

Fuente: Elaboración propia.

Como conclusión, podemos resaltar que los mayores porcentajes de todos los tramos de edades se concentran en las frecuencias de 0 a 4 veces al año en visitas a los museos que componen la muestra. Siendo los menores de 44 años los que más asistencia acumulan en esta franja. Por el contrario, los mayores de 45 años $(60,4 \%)$ afirman visitar más de 8 veces al año.

\subsection{Valoración de los recursos digitales en función de la edad del visitante}

Teniendo en cuenta la valoración de los recursos digitales ofertados por los museos de la muestra, observamos que el grupo de más de 45 años (42,5\%) es el que más/con mayor prevalencia afirma estar de acuerdo con solo usar la página web del Museo para buscar informaciones prácticas (horarios, tarifas, reservas), seguidos de la franja de edades entre 25-44 años (37,0\%), y los menores de 24 años (35,2\%). También destaca la indiferencia del grupo menor de 24 años respecto a esta afirmación con un porcentaje del $39,8 \%$, seguido de la franja 25-44 años (37,0\%), y por último, el grupo mayor de 45 años $(31,1 \%)$.

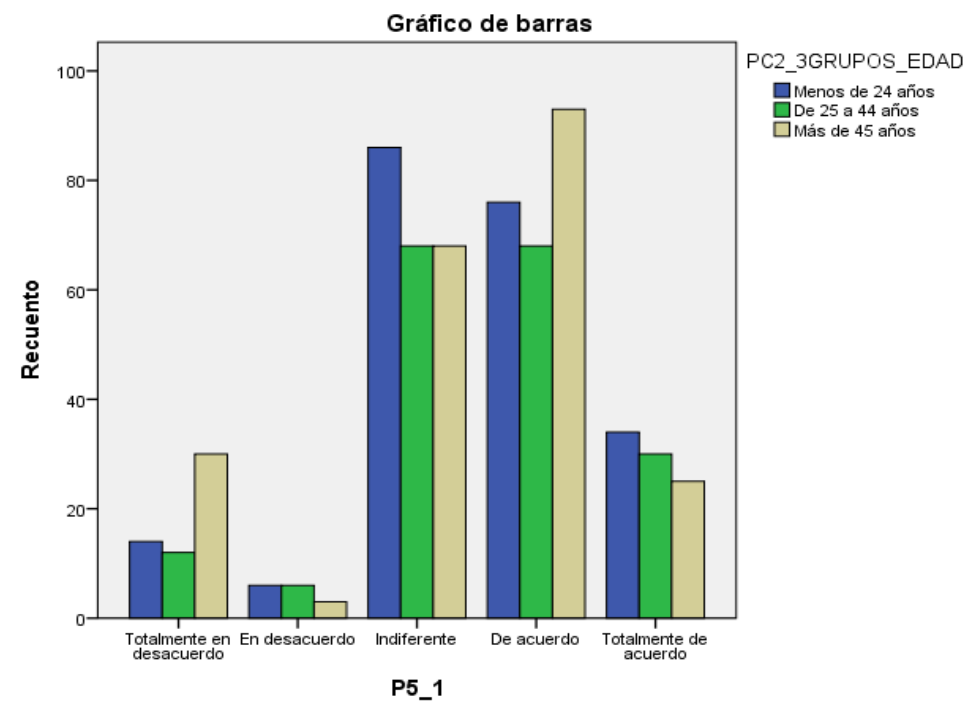


Gráfico 5: Histograma de la variable sólo usar la página web del Museo para buscar informaciones prácticas (horarios, tarifas, reservas).

Fuente: Elaboración propia.

Por su parte, el 47,5\% de los mayores de 45 años afirman que en la página web busco informaciones sobre la colección e imágenes de las obras, seguidos por los menores de 24 años $(26,9 \%)$ y del grupo de $25-44$ años (26,1\%). En esta ocasión, es la franja entre $25-44$ años $(43,5 \%)$ los que muestran una mayor indiferencia, en segundo lugar los menores de 24 años (38,9\%), y en tercer lugar la franja de más de 45 años $(32,4 \%)$.

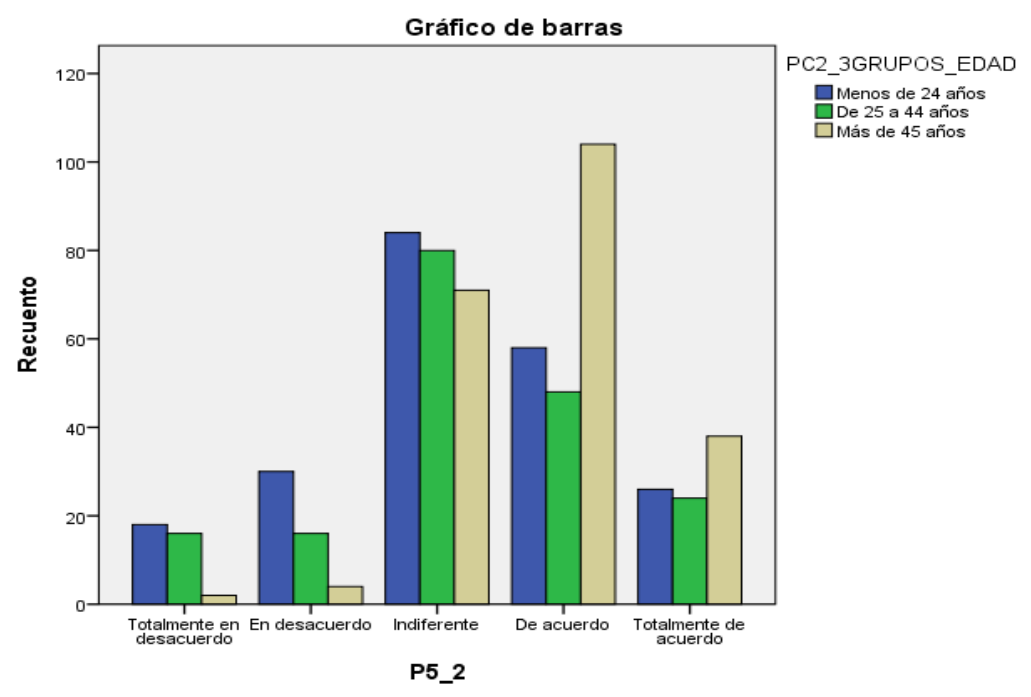

Gráfico 6: Histograma de la variable en la página web busco informaciones sobre la colección e imágenes de las obras.

Fuente: Elaboración propia.

En resumen, a menor edad mayor interés por los contenidos interactivos de la página web de los museos. Sin embargo, son las franjas de edad mayores (45 años en adelante) los que muestran un mayor interés a la hora de valorar la página web, los contenidos y la información propuesta. Siendo los menores de 24 años los que mayor indiferencia muestran hacia los recursos web de los museos de la muestra.

A la hora de valorar las redes sociales, el 42,5\% de los visitantes menores de 24 años afirman no seguir a menudo información sobre el Museo en las redes sociales, en segundo lugar, se encuentran los visitantes entre 25-44 años (32,6\%) y, por último, los mayores de 45 años (21\%). Es decir, el grupo mayor de 45 años es el que muestra un mayor seguimiento de las redes sociales del museo que visita, y el grupo entre 25-44 años los que mayor indiferencia muestran (42,4\%). En concreto, para el 69,5\% (de acuerdo y totalmente de acuerdo) de los menores de 24 años las redes sociales permiten crear una interacción con los museos que no existía antes, seguidos del grupo entre 25-44 años (68,5\%), y de los mayores de 45 años (65,3\%). La indiferencia mostrada en esta pregunta aumenta al aumentar la edad del visitante. 


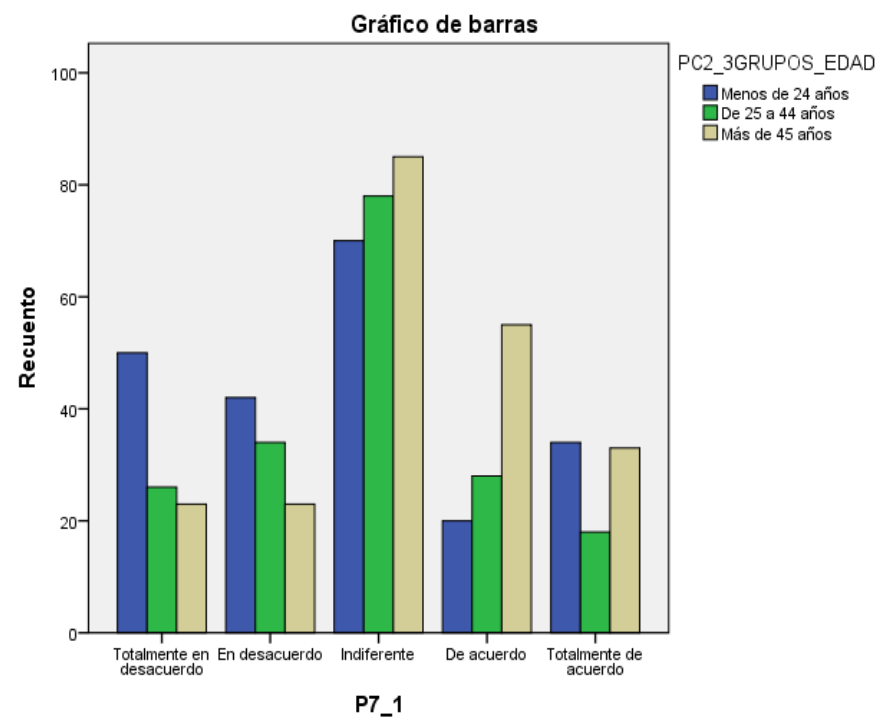

Gráfico 7: Histograma de la variable no sigo a menudo información sobre el Museo en las redes sociales.

Fuente: Elaboración propia.

Se puede concluir que los menores de 24 años son los que menos siguen las redes sociales de los museos que visitan, aunque si valoran positivamente el uso de las redes sociales y la interactividad. Los mayores de 45 años son los que siguen mayor medida los perfiles sociales y los que mayor relevancia otorgan a las redes de los museos.

Teniendo en cuenta la edad en la valoración de las visitas virtuales, se detecta que el 39,3\% de los mayores de 45 años se muestran de acuerdo con que el Museo que visito explota y aprovecha al máximo la visita virtual. Por el contrario, los otros dos grupos de edad presentan mayor prevalencia en la respuesta en desacuerdo. En concreto, el $32,6 \%$ de los visitantes entre $25-44$ años y el $24,1 \%$ de los menores de 24 años creen que los museos que visitan no aprovechan las visitas virtuales. Además, en cuanto a la indiferencia mostrada, todos los grupos destacan por obtener elevadas frecuencias en esta respuesta. 


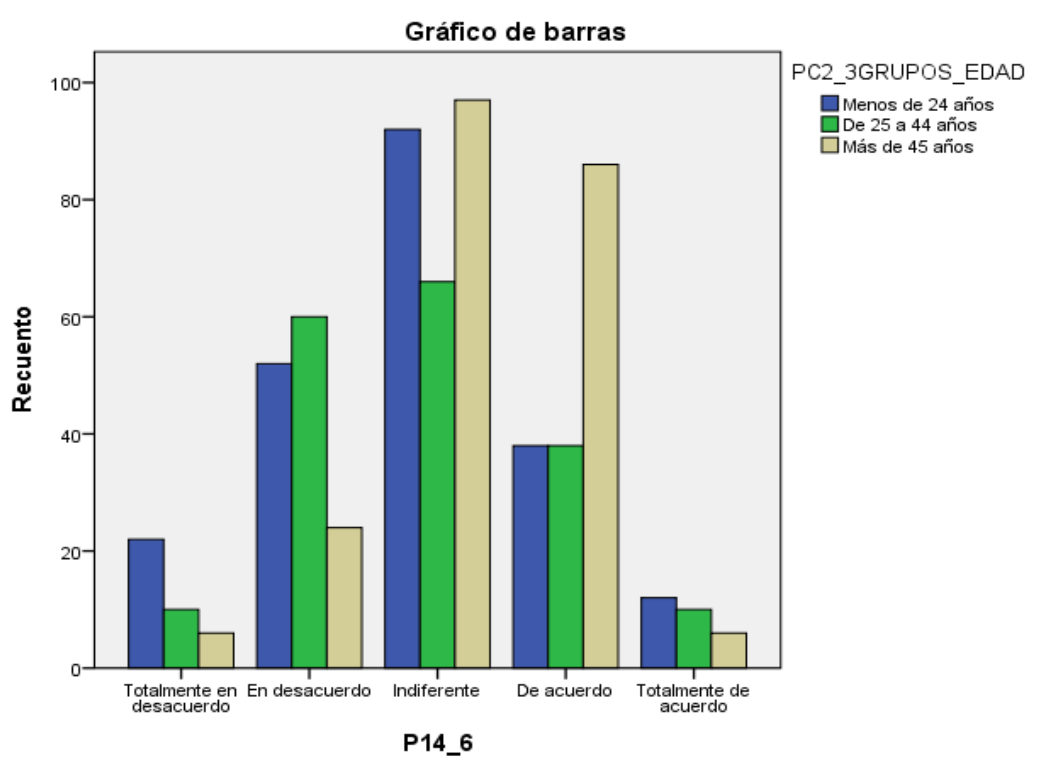

Gráfico 8: Histograma de la variable el Museo que visito explota y aprovecha al máximo la visita virtual.

Fuente: Elaboración propia.

Estos resultados permiten observar que los visitantes más jóvenes son más críticos con el uso que hacen los museos de las visitas virtuales. Además, los visitantes más mayores se muestran de acuerdo con la importancia de las visitas virtuales y su apoyo durante la visita.

\subsection{Valoración del grado de aprovechamiento personal y aprendizaje durante la visita en función de la edad}

El análisis de la valoración del aprendizaje durante la visita por edad permite observar varios aspectos interesantes. Para el 54,6\% de los visitantes con menos de 24 años, las aplicaciones digitales son imprescindibles para los museos de hoy en día, el $46,6 \%$ de los visitantes mayores de 45 años, también se muestran de acuerdo, y en último lugar, el grupo de edad entre 25-44 años (39,1\%). Observando los resultados, a mayor edad mayor prevalencia en la respuesta indiferente. 


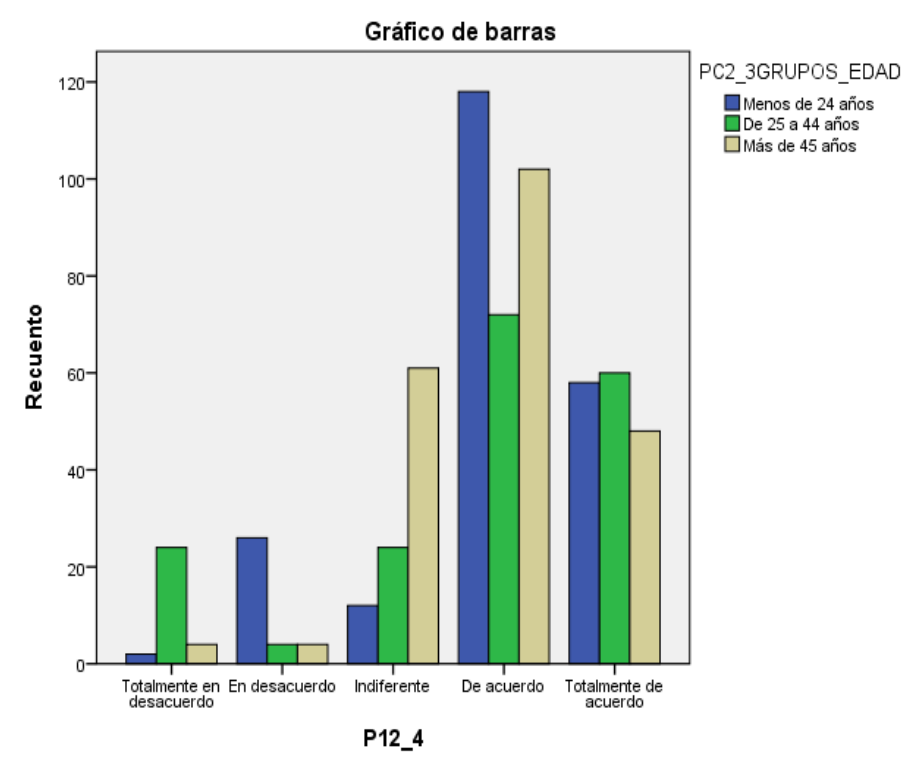

Gráfico 9: Histograma de la variable las aplicaciones digitales son imprescindibles para los museos de hoy en día.

Fuente: Elaboración propia.

El 48,9\% de los visitantes mayores de 45 años se muestran en desacuerdo con la pregunta: Un museo como el que visito no necesita desarrollar aplicaciones digitales como las Apps. El segundo grupo de visitantes en mostrar su desacuerdo son los menores de 24 años (39,8\%), y finalmente la franja entre 25-44 años (32,6\%). La indiferencia aumenta al aumentar la edad del visitante.

Al 49,3\% del público mayor de 45 años le gustaría que las Apps del Museo propongan juegos y recorridos divertidos y lúdicos, seguidos de los visitantes entre $25-44$ años (42,4\%) y de los menores de 24 años (40,3\%). En cuanto a la respuesta indiferente, los porcentajes aumentan al aumentar la edad del visitante.

En definitiva, los grupos de edades más jóvenes creen que las aplicaciones son imprescindibles para los museos y a mayor edad, mayor creencia de la necesidad de las aplicaciones en museos para fomentar el aprendizaje. Aunque en todas las preguntas los grupos de edad mayores tienden a ser más indiferentes en las respuestas.

\section{DISCUSIÓN Y CONCLUSIONES}

Los resultados demuestran que, el público menor de 24 años es el segundo grupo de visitantes que visita con mayor asiduidad los museos y en sus respuestas a los cuestionarios se manifiestan diciendo que las visitas a los museos virtuales complementan de manera divulgativa a la visita física, pero a la vez, es el grupo de edad que menor interés muestra por los contenidos web y redes sociales de los museos que visitan, seguidos de los adultos jóvenes, sujetos de 25 a 44 años. Asimismo, los visitantes jóvenes destacan por el uso de dispositivos móviles durante la visita para hacer fotografías y utilizar sus propias redes sociales, parece que las redes del museo no captan su atención, según los resultados. 
Esta constatación nos lleva a la argumentación de que el presunto desencanto que se les atribuye a los jóvenes por el museo ocultaría la incomprensión del papel que juegan las nuevas tecnologías, lo que influye en la presencia del museo en la sociedad contemporánea. Las iniciativas conservadoras de los museos para promover el acceso de los jóvenes pasan por la oferta de descuentos, mientras que las estrategias más vanguardistas son aquellas que buscan ejecutar estrategias integrales para fortalecer el vínculo con los jóvenes reconociendo, en primer lugar, sus necesidades (AntoineFaúndezy Carmona-Jímenez, 2014). Tal vez sería conveniente desarrollar esquemas de igualdad (Jones, 2012) que permitan crear categorías específicas con beneficios asociados a un perfil de visitante joven como el Young Friends del Philadelphia Museum of Art -grupo de amigos del museo entre 21 y 44 años con intereses comunes, con descuentos en eventos, clases gratuitas e invitaciones a programas -, o la estrategia seguida por el Metropolitan Museum de Nueva York que supera el descuento tradicional en la entrada, ofreciendo una gran variedad de ventajas por solo ser joven, como guías audiovisuales gratuitas o pasantías remuneradas para estudiantes de secundaria. Estas estrategias suponen un esfuerzo económico para los museos, pero facilitaría la construcción de una relación de sentido entre los jóvenes y el museo, superando de esta forma, la barrera de comunicación con un público a priori desconectado.

Otro punto a destacar, relacionado con el escaso interés por los contenidos web de los museos que muestran los visitantes más jóvenes, sería el establecimiento de las particularidades del público joven, identificando sus necesidades y de acuerdo a ello ofrecer contenidos, servicios y actividades dirigidas, en especial, a estimular la participación de los jóvenes con el medio digital de la entidad cultural. Esto es, no basar las experiencias de nexo entre museo y jóvenes exclusivamente en talleres y visitas familiares sin tener en cuenta las singularidades que la caracterizan como grupo multigeneracional.

Por otro lado, aunque el grupo de visitantes menor de 24 destaca por el uso de dispositivos móviles durante la visita, no son los que mayor seguimiento presentan tanto de redes sociales como de interacción digital con el museo. Es importante resaltar que, aunque el museo cuente con recursos digitales como tabletas, realidad virtual o aumentada e interactivos, lo más importante es que un museo se adapte al tipo de público a través de la predisposición a interactuar con ellos, cambiando desde una exposición pasiva a un ente vivo (Santacana et al., 2016). En relación a las redes sociales del museo, se propone aprovechar las nuevas tecnologías de la información y la comunicación para conferir al público joven un papel más activo en el proceso de la comunicación del museo. Utilizar la web, el blog o el Facebook de jóvenes de los museos sería una oportunidad para que el museo se dirija a los jóvenes informando sobre lo que hace, pero a la vez usando estas plataformas como un espejo que refleja lo que éstos opinan y dialogan sobre el museo.

Asimismo, los datos permiten comprobar cómo el público visitante de los museos de la muestra se caracteriza por realizar fotografías, indicar su ubicación, compartir

Vivat Academia. Revista de Comunicación. 2021, nº 154, 21-43 
fotografías y buscar información en Internet durante la visita. Además, son los hombres los que mayores búsquedas en Internet realizan durante la visita y cuanto mayor es el nivel de estudios, mayor es la frecuencia de búsqueda de información. Por otra parte, aunque el público en general le otorga importancia al uso de las redes sociales y su interactividad, los visitantes mayores de 45 años son los que siguen con mayor frecuencia los perfiles sociales de los museos que visitan. Además, el nivel de estudios también influye en el uso de dispositivos y el uso de redes sociales, a mayor nivel de estudios mayores porcentajes de utilización.

Estas reflexiones nos llevan al contexto actual caracterizado por la aparición de nuevos hábitos de consumo de información y que está modificando la forma en que los ciudadanos y, por ende, la audiencia de los museos, atribuyen relevancia a la actualidad (Casero-Ripollés, 2020). Como se puede observar en los resultados de esta investigación, está creciendo el número de visitantes que acceden a la información a través de redes sociales y servicios móviles, lo que implica un cambio en la forma de informarse.

Esta tendencia en la búsqueda de información y uso de redes sociales durante la visita puede ser una gran ventaja o desventaja para los museos, si éstos no superan los dos problemas que afectan el escenario mediático actual; la saturación y el desinterés de la audiencia. En la práctica habría que trabajar teniendo en cuenta que los criterios de los ciudadanos cambian porque la audiencia es cada vez más exigente y crítica (Echegaray-Eizaguirre, 2015). Tanto los intereses como los comportamientos evolucionan de manera constante y ya no se conforman con las ofertas tradicionales. Esta audiencia busca contenidos especializados y novedosos adaptados a su forma de vida. Por tanto, la audiencia de los museos responde a un perfil muy diferente al que caracterizaba a las generaciones pasadas, y determinan el consumo de información y las acciones realizadas durante la visita a los museos.

Gracias a Internet y a las redes sociales, el público de los museos dispone de una nueva forma de relacionarse con el espacio y gestionar su tiempo durante la visita, $y$ este hecho afecta a las formas de acceso a los contenidos y al consumo de información en los museos. En este sentido, los museos deberían trabajar utilizando varias estrategias que permitan un consumo de contenidos al nivel que cabría esperar el público en función de sus intereses inmediatos y futuros, y que repercutiría positivamente en la imagen que la audiencia tiene sobre el ente cultural.

Es de interés conocer la valoración de los recursos digitales que ofrece el museo, para poder implementar mejoras o subsanar iniciativas que no estén obteniendo el impacto requerido. Tomando como referencia al público de este estudio, según los resultados aquellos visitantes con mayor nivel de estudios y de 25 años en adelante muestran un mayor interés por las visitas virtuales en los museos. Podemos decir que, las visitas virtuales es uno de los interactivos que mayor atención parece, en un primer momento, atraer al público-audiencia de los museos, pero para que estas tecnologías sean un valor de atracción deben cumplir con las expectativas de los consumidores. Por consiguiente, analizar a grandes entes culturales que están llevando a cabo

Vivat Academia. Revista de Comunicación. 2021, nº 154, 21-43 
iniciativas relacionadas con la realidad virtual como el Metropolitan Museum de Nueva York, El Louvre y el Museo del Prado, entre otros, sería un punto de partida para readaptarse a las nuevas corrientes digitales lideradas por estos museos.

Al valorar las visitas virtuales la mayoría del público visitante se mostraba de acuerdo en que eran valiosas, complementaban la visita física y acercaban al museo. Sin embargo, en la práctica los museos de la muestra no contaban con una visita virtual adaptada a las necesidades de la audiencia actual. Esta situación, se ha revertido en la primera mitad del año 2020 como consecuencia directa de la llegada del COVID-19, y hemos podido asistir al impulso de estrategias digitales por los museos para seguir conectados con sus públicos, que pueden ser el germen de posteriores estrategias digitales.

Siguiendo este hilo argumental, el Museo del Louvre anunció -provocado por el cierre indefinido del COVID-19 -la habilitación de recorridos virtuales en 360 grados para que los interesados en visitar este espacio puedan hacerlo desde sus casas. Por su parte, el Metropolitan Museum de Nueva York ofrece la posibilidad de sumergirse en una serie de seis vídeos en 360 grados para experimentar "la magia de pararse en una galería vacía después de horas, presenciar un espacio bullicioso en el lapso de tiempo, o flotar por encima de The Met Cloisters para una vista panorámica" (MET, 2020). Estos vídeos están disponibles en YouTube para Smartphone, ordenador y Google Cardboard o auriculares VR.

En España, el Museo del Prado pone a disposición una colección virtual a través de su página web con más de 18.000 obras. En cada una de ellas puedes encontrar el autor, descripción y ficha técnica; e incluso permite colocar las piezas en una línea de tiempo para ver la evolución pictórica. Además, toda la información puede encontrarse en el canal de YouTube del museo.

La pandemia mundial puede ser un momento crucial, y de esfuerzo económico, para captar y mantener tanto al público joven como aquel público más desconectado de este tipo de experiencia museística. Pero ¿qué hacen los museos de la muestra? Por un lado, el Museo de la Evolución Humana de Burgos ha puesto en marcha un recorrido virtual, que hasta ese momento no disponía, por 21 espacios del museo explicados por su coordinadora, Aurora Martín y el responsable de didáctica, Rodrigo Alonso. Por otro lado, el Museo Casa Lis de Salamanca, que ya contaba con una visita virtual a través de Quicktime, con el cierre temporal del museo comparte contenido, juegos, actividades y concursos a modo de ventana virtual a través de la web, Facebook, Twitter e Instagram.

Estas diferencias entre unos museos que ya cuentan con un largo recorrido de tecnología al servicio de la ciudadanía y que han podido seguir manteniendo una comunicación y proximidad con sus públicos en tiempos de pandemia, nos lleva a reflexionar sobre la necesidad de gestionar los recursos digitales de forma eficaz y estable en el tiempo. Supone un esfuerzo económico, pero facilitaría la fluidez en la comunicación y evitaría la pérdida de visitantes potenciales en tiempos de necesidad.

Vivat Academia. Revista de Comunicación. 2021, n 154, 21-43 
El objetivo es, por tanto, ayudar a construir en hábito de uso y consumo, incluso acostumbrar a los ciudadanos a que paseen virtualmente por los museos y evitar que una pandemia fracture los mecanismos de comunicación e interacción entre el museo y sus visitantes. Los resultados de nuestra investigación y la contextualización del catalizador tecnológico del COVID-19, contextualizan y certifican una aceleración del cambio de paradigma comunicativo y educativo que ya existía en proceso en los museos de nuestro contexto sociocultural. El vértigo de los espacios culturales, de las industrias culturales sigue siendo el de adaptarse de forma proactiva a las demandas de una sociedad en constantes cambios y de una audiencia fragmentada, segmentada y en plena revolución. Las oportunidades comunicativas y educativas son inmensas para nuestros museos físicos y digitales, esos espacios que son ahora llamados a ser también los espacios virtuales a golpe de clic de una audiencia global cada vez más ávida del mejor entretenimiento y contenido cultural, también museístico.

\section{REFERENCIAS}

Almazán-Fernández de Bobadilla, L. (2007). Dimensión educativa de los centros de arte: investigación basada en usuarios del Centro José Guerrero de Granada [Tesis Doctoral, Universidad de Granada]. Repositorio Institucional: http://hdl.handle.net/10481/1473

American Alliance of Museums, AAM (2020a). 4 ways museums can successfully leverage digital content and channels during Coronavirus (COVID-19). https://www.aam-us.org/2020/03/25/4ways-museums-can-successfullyleverage-digital-content-and-channelsduring-coronavirus-COVID-19/

American Alliance of Museums, AAM (2020b). How your museum can use social media during COVID-19. https://www.aam-us.org/2020/03/24/how-yourmuseum-can-use-social-media-during-COVID-19/

Antoine-Faúndez, C. D. y Carmona, J. J. (2014). Museos y jóvenes. Entre la incomprensión y el desencanto. Percepciones y argumentos juveniles sobre el consumo cultural de museos en Chile. Arte, Individuo y Sociedad, 27 (2), pp. 259-274. ISSN: 1131-5598. http://dx.doi.org/10.5209/rev_ARIS.2015.v27.n2.44561

Ayala-Aizpuru, I., Cuenca-Amigo, M. y Cuenca-Amigo, J. (2019). Principales retos de los museos de arte en España. Consideraciones desde la museología crítica y el desarrollo de audiencias. Aposta. Revista de Ciencias Sociales, 80, pp. 61-81. http://apostadigital.com/revistav3/hemeroteca/iayala.pdf

Black, G. (2012). Transforming Museums in the Twenty-first Century. Nueva York: Routledge. ISBN: 1136515771, 9781136515774 
Casero-Ripollés, A. (2020). Impact of COVID-19 on the media system. Communicative and democratic consequences of news consumption during the outbreak. El $\begin{array}{lllll}\text { profesional de la información, } 29 & \text { (2), e290223. }\end{array}$ https://doi.org/10.3145/epi.2020.mar.23

Cordón-Benito, D. (2012). El presente de los museos, retos y oportunidades a las que enfrentarse. Capital Humano: Revista para la integración y desarrollo de los recursos humanos, 25 (269), pp. 46-49.

Delgado, J. C. (2018). Fundación Thyssen Bornemisza: el guardián de un tesoro nacional. ABC, 13 de febrero. https://www.abc.es/cultura/arte/abci-fundacion-coleccionthyssen-bornemisza-guardian-tesoro-nacional-201802110124_noticia.html

Delgado Pacheco, S. (2020). Los retos en investigación, comunicación y educación de los museos de Lima en el marco de la COVID-19. Una discusión sobre los desafíos del futuro a partir de su situación actual y de las experiencias del MALI y el MUCEN. Desde el Sur, 12(1), 285-306. http://dx.doi.org/10.21142/des-1201-2020$\underline{0017}$

Echegaray-Eizaguirre, L. (2015). Los nuevos roles del usuario - audiencia en el entorno comunicacional de las redes sociales. En Quintas-Froufe y González-Neira (coord.), La participación de la audiencia en la televisión de la audiencia activa a la social (pp. 2746). Madrid. Asociación para la Investigación de Medios de Comunicación (AIMC). ISBN 978-84-608-4242-2. http://hdl.handle.net/2183/16235

Holmquist, U. (2020). El British Council y los museos peruanos: colaboraciones para la sostenibilidad $y$ resiliencia. https://pe.live.solas.britishcouncil.digital/sites/default/files/el_british_council_ y_los_museos_peruanos.pdf

ICOM. (2020). Cómo comunicarse a distancia con su público. Consejo Internacional de Museos. https://icom.museum/es/COVID-19/recursos/como-comunicarse-adistancia-con-su-publico/

Jones, P. y MacLeod, S. (2016). Museum architecture matters. Museum and Society, 14 (1), pp. 207-219. https://doi.org/10.29311/mas.v14i1.635

Juste, M. y Fernández, J. (2016). Así utilizan las tecnologías los museos españoles. Expansión, 14 de febrero. https://www.expansion.com/economiadigital/innovacion/2016/02/12/56bdca8 $\underline{822601 \mathrm{~d} 4 \mathrm{~b} 438 \mathrm{~b} 45 \mathrm{ab} \cdot \mathrm{html}}$

Lorente, J. P. (2015). Estrategias museográficas actuales relacionadas con la museología crítica. Complutum, $26 \quad$ (2), pp. 111-120. https://doi.org/10.5209/rev_CMPL.2015.v26.n2.50422 
Marakos, P. (2014). Museums and social media: Modern methods of reaching a wider audience. Mediterranean Archaeology y Archaeometry, 14 (4), pp. 75-81. http://maajournal.com/Issues/2014/Vol14-4/Full9.pdf

Mariátegui, J. (2020). Día Internacional de los Museos: ¿Cómo se transformarán digital y culturalmente? El Comercio, 17 de mayo. https://elcomercio.pe/eldominical/actualidad/lanuevanormalidadtransformacion-digital-y-cultural-en-los-museos-noticia/

Mateos-Rusillo, S. (2012). Manual de comunicación para museos y atractivos patrimoniales. Ediciones Trea.

Perez-Rufi, J. P. (2020). La comunicación audiovisual durante la crisis sanitaria del coronavirus COVID-19. https://hdl.handle.net/10630/19691

Quero, M. J. y Leal-Jiménez, A. (2011). Manual de marketing y comunicación cultural. Dirección General de Universidades de la Consejería de Economía, Innovación y Ciencia de la Junta de Andalucía. Servicio de publicaciones de la Universidad de Cádiz, Cádiz. http://uprid2.up.ac.pa:8080/xmlui/handle/123456789/1620

Riofrío Flores, M. D. P., Alvarado Peña, E., Cueva Chacón, P. y Guerra Chirinos, D. (2019). Agenda de sostenibilidad para los museos y centros expositivos de Lima: ruta para su incidencia en el desarrollo sostenible de la ciudad. Lima: Museo de Arte de Lima.

Rodríguez-Frade, J. P. (2011). Reflexiones sobre museografía sostenible. Museos.es, 7 (8), pp. 182-189. https://sede.educacion.gob.es/publiventa/reflexiones-sobremuseografia-sostenible/museos/20919C

Santacana, J., Martínez-Gil, T., Llonch-Molina, N. y López-Benito, V. (2016). ¿Qué opinan los adolescentes sobre los museos y la didáctica? Didáctica de las Ciencias Experimentales y Sociales, 31, pp. 23-38. http://hdl.handle.net/2445/155826

Tamacas, C. M. (2016). Usos de la tecnología móvil en los museos de Nueva York. kóot, 7, pp. 9-14. http://biblioteca.utec.edu.sv/sitios/koot/index.php/koot/article/view/84

The Metropolitan Museum of Art. (2020). Exhibitions. https://www.metmuseum.org/

\section{AUTORES}

\section{Inma García Martín}

Universidad de Salamanca (USAL). España. Doctoranda en el Programa de Doctorado en Formación en la Sociedad del Conocimiento de la Universidad de Salamanca. Graduada en Comunicación Audiovisual y Máster en Evaluación y Gestión del 
Patrimonio Cultural por la Universidad de Salamanca, actualmente se encuentra realizando su Tesis Doctoral titulada "Hábitos de Uso y Consumo Educomunicativos en los Espacios Museísticos de Castilla y León. Impacto de las aplicaciones digitales en el público de los museos". Sus líneas de investigación son comunicación, educación, aplicaciones digitales, audiencias, hábitos de uso y consumo en museos.

adagarcia@usal.es

\section{Índice $\mathrm{H}: 1$}

Orcid ID: https:// orcid.org/0000-0003-3467-6802

Google Scholar: https://scholar.google.es/citations?user=yNsC-owAAAAJ\&hl=es

\section{Félix Ortega Mohedano}

Universidad de Salamanca (USAL). España. Profesor Contratado Doctor del Departamento de Sociología y Comunicación Audiovisual de la Universidad de Salamanca (Usal). Profesor del Doctorado en Formación en la Sociedad del conocimiento - http://knowledgesociety.usal.es/ - y Director del Master en Comunicación. Investigación e Innovación, - http://mucaii.usal.es/-, Universidad de Salamanca, Asignaturas: Los Mass Media ante la Sociedad del Conocimiento y la Creatividad, Economía Digital, Comunicación y Educación, Profesor del Grado en Comunicación Audiovisual, Asignaturas Investigación de Audiencias, Programación en las Industrias Culturales y Audiovisuales, Comunicación y Educación.

fortega@usal.es

\section{Índice $\mathrm{H}: 10$}

Orcid ID: https:// orcid.org/0000-0003-2735-4813

Google Scholar: https://scholar.google.es/citations?user=qS0FrGoAAAAJ\&hl=es

\section{María Esther Pérez Peláez}

Universidad Internacional de Valencia (VIU). Profesora de la Universidad Internacional de Valencia (VIU), en la Facultad de Educación. Ha sido Coordinadora Académica y profesora del MBA en Empresas e Instituciones Culturales de la Universidad de Salamanca, Colabora como investigadora con la Universidad de Salamanca, la Universidad Rey Juan Carlos, la Cátedra UNESCO Iberoamericana de Danza "Alicia Alonso" y la Cátedra UNESCO UNITWIN de centros superiores de artes escénicas.

mariaesther.perez@campusviu.es

\section{Índice $\mathrm{H}: 1$}

Orcid ID: https:/ / orcid.org/0000-0001-5243-5733

Google Scholar: http://cort.as/-HUhB 